\title{
Inter-operator reliability of live football match statistics from OPTA Sportsdata
}

\author{
Hongyou Liu ${ }^{1,2}$, Will Hopkins ${ }^{3}$, Miguel A. Gómez ${ }^{1}$ and Javier S. Molinuevo ${ }^{1}$ \\ ${ }^{1}$ Faculty of Physical Activity and Sport Sciences, Polytechnic University of Madrid, \\ Madrid, Spain \\ ${ }^{2}$ Sports Department, Shenzhen University, Shenzhen, China \\ ${ }^{3}$ School of Sport and Recreation, Auckland University of Technology, Auckland, New \\ Zealand
}

\begin{abstract}
The aim of the study was to evaluate the inter-operator reliability of OPTA Client System which is used to collect live football match statistics by OPTA Sportsdata Company. Two groups of experienced operators were required to analyze a Spanish league match independently. Results showed that team events coded by independent operators reached a very good agreement (kappa values were 0.92 and 0.94) and average difference of event time was $0.06 \pm 0.04$ s. The reliability of goalkeeper actions was also at high level, kappa values were 0.92 and 0.86 . The high intra-class correlation coefficients (ranged from 0.88 to 1.00) and low standardized typical errors (varied from 0.00 to 0.37 ) of different match actions and indicators of individual outfield players showed a high level of inter-operator reliability as well. These results suggest that the OPTA Client System is reliable to be used to collect live football match statistics by well trained operators.
\end{abstract}

Keywords: Reliability, football, match statistics, performance indicators, OPTA Sportsdata.

\section{Introduction}

Reliability refers to the consistency, repeatability or reproducibility of values, scores, data or outcomes of a test, assessment, assay or other measurements in repeated trials on 
the same individuals (Hopkins, 2000a; Downing, 2004; Berg and Richard, 2008).

The importance of reliability assessments in sport performance analysis has been widely discussed (Hughes et al., 2001; Hughes et al., 2004; Valter et al, 2006; Bradley et al., 2007; Lames and McGarry, 2007; O’Donoghue, 2007; Robinson and O'Donoghue, 2007; O'Donoghue, 2010). It is believed that reliability evaluation could provide information to interpret the understanding of measurement error involved in performance analysis (O’Donoghue, 2007).

Most performance analysis methods involve manual data collecting techniques whose reliability is limited due to human errors (O'Donoghue, 2007). Measurements in performance analysis should be reproducible over different observers or operators, because poor reproducibility limits the ability of data users to reach the conclusions or objectives with the measured variables (Hayen et al. 2007). Therefore, a reliability test in this kind of data gathering methods is of much importance.

Previously, lots of studies focusing in football performance analysis had used different sources of data, mainly included Prozone Sports Ltd $^{\circledR}$ (Valter et al, 2006; Bradley et al., 2007; Bradley et al., 2011), Gecasport (Gómez et al., 2009a; 2009b; 2012; Lago-Peñas et al., 2010), Amisco Pro ${ }^{\circledR}$ (Di Salvo et al, 2007; Carling et al., 2008; Lago et al., 2010; Dellal et al., 2011) and OPTA Sportsdata (Oberstone, 2009; 2010; 2011). The accuracy and reliability of former three data resources have already been verified, while the data from OPTA Sportsdata has still not been validated.

OPTA Sportsdata is a company founded in 1996 in London, which dedicated for years in collecting, compiling, analyzing, storing, distributing and supplying live sports data on a wide variety of sports across the world. Although the company provides live and archive data services in up to 40 sports, such as cricket, tennis, rugby, handball, its expertise focuses on football. Football match performance data from the company has been widely used in the betting industry, broadcasting, online and mobile media, print publishers, sponsors and brands, professional clubs and governing bodies (Optasports) ${ }^{1}$.

As mentioned above, for the statistics provided by the company to be used validly, their data collecting methods should be reliable (Bradley et al., 2007). This issue is not only important in academic research of performance analysis, but also in public using and coaching purposes (Bradley et al., 2007; O’Donoghue, 2007).

Cooperating with OPTA Spain (Madrid), the current study aimed to determine the inter-operator reliability of OPTA Client System which is used to collect live football match statistics by the company when operated by different well trained operators.

\footnotetext{
${ }^{1}$ http://www.optasports.com/
} 


\section{Methods}

\subsection{Software and Data Entry}

The software used by OPTA Sportsdata is the OPTA Client System which can be used to generate live match statistics for any match covered in the SSTZ game system.

Every possible type of ball touch and on the ball actions in match is covered by a rigid set of definitions (see section 2.2) which are recorded in the system. The analysts are undergone a strict training to learn thoroughly about the definitions and to get familiar with the shortcut keys of different actions in the system (see Figure 1) before formally operating. During the training, an analyst is allowed to use a "test servers" to imitate a live match situation (OPTA, 2012).



Figure 1. Shortcut Keys of Different Actions in the OPTA Client System

In the formal operation, the analyst has the full responsibility to interpret what he chooses and logs the action using one of the definitions. Every action requires a player to be assigned to it, along with a time (mm:ss of match time) showing when the event actually happened. For events to be matched, the maximum time on the timeline observations could differ by is limited to 2 seconds. To highlight a player, number keys responding to the player's shirt numbers are used to differentiate between players. It will need two operators in one match, one for home team and the other for away team 
(OPTA, 2012).

All the players' ball touch and on the ball actions and their happening time can be recorded and output by the system. The team actions (except "ball possession") are the sum of all individual players. The ball possession is calculated by the time of ball control by players from different teams.

\subsection{Operational Definitions of Match Actions}

Any match actions or variables that have not been defined need to be defined precisely which makes their meaning unambiguous (O'Donoghue, 2007; 2010). It is also important for the actions to have a metric property to be well interpreted and coded by operators (O'Donoghue, 2006).

Following are the operational definitions of main match actions drawn from OPTA (Bateman, 2010; OPTA, 2012).

Actions of outfield players:

Aerial Duel: Two players competing for a ball in the air, for it to be an aerial both players must jump and challenge each other in the air and have both feet off the ground. The player who wins the duel gets the Aerial Won, and the player who doesn't gets an Aerial Lost.

Assist: The final pass or cross leading to the recipient of the ball scoring a goal.

Ball recovery: The event given at the start of a team's recovery of possession from open play. In order to give a ball recovery the defending team must have full control of the ball and must start a new passage of play.

Block: A defensive block, blocking a shot going on target. This must be awarded to the player who blocks the shot.

Challenge: A defender tries to stop a player dribbling past him. The player who has been beaten is given a challenge lost if he does not win the ball.

Clearance: Players attempt to get the ball out of the danger zone, when there is pressure behind them, or there is pressure on the player to clear the ball from the danger zone.

Cross: Any ball played into the opposition team's area from a wide position.

Dispossessed: When a player is tackled without attempting to dribble past his opponent. 
Dribble: An attempt by a player to beat an opponent in possession of the ball. A successful dribble means the player beats the defender while retaining possession; unsuccessful ones are where the dribbler is tackled. OPTA also log attempted dribbles where the player overruns the ball.

Foul: Any infringement that is penalised as foul play by a referee.

Interception: An interception is given when a player intercepts a pass with some movement or reading of the play. In order to give an interception, the player must read the pass and move his body to cut the pass out.

Key Pass: The final pass or cross leading to the recipient of the ball having an attempt at goal without scoring.

Offside: Being caught in an offside position resulting in a free kick to the opposing team.

Pass: An intentional played ball from one player to another.

Shot: An attempt to score a goal, made with any (legal) part of the body, either on or off target. The outcomes of shot could be: goal, shot on target, shot off target, blocked shot, post.

Tackle: The act of gaining possession from an opposition player, when he is in possession of the ball. A tackle won is given when a player makes a tackle and possession is retained by either himself or one of his team mates. Tackle won was also given when the tackle results in the ball leaving the field of play.

Through Ball: A pass playing a player through on goal which could lead to a goal scoring opportunity. The pass needs to split the last line of defence and plays the teammate through on goal.

Touches: A sum of all events where a player touches the ball, so excludes things like aerial lost lost or challenge lost.

Turnover: Loss of possession due to a mistake/poor control.

Actions of goalkeepers:

Catch: The goalkeeper catching a cross or a ball played in to the area when there is pressure from an opposition player asserted on him.

Collected Ball: The goalkeeper picking up the ball from open play in the box. 
Cross not Claimed: The goalkeeper attempting to claim a cross, but making no contact with the ball.

Drop: When the goalkeeper goes to claim a cross, and somewhat gathers the ball then manages to drop it.

Goalkeeper Kick from Hands: The goalkeeper kicks the ball from his hands after picking the ball up.

Goalkeeper Launch: When the goalkeeper has the ball in his hands and he puts the ball down to kick it long up the field.

Goalkeeper Throw: The Goalkeeper throwing the ball out to a player after he picks it up.

Keeper Sweeper: When the goalkeeper runs out from the goal line to either intercept a pass or close down an attacking player.

Penalty Faced: Given when the goalkeeper is facing a penalty

Punch: The goalkeeper punches a cross or a ball played into the box.

Save: The goalkeeper prevents the ball from entering the goal with any part of his body.

Smother: The goalkeeper makes a tackle when an opposition attacking playing is trying to dribble around him.

\subsection{Data Collection}

The match of first round of Spanish La Liga BBVA 2012-2013 between Real Madrid and Valencia held on $19^{\text {th }}$ August, 2012 was chosen as the subject of the current inter-operator reliability study. In total, 28 players were involved, including 2 goalkeepers and 6 substitutions.

Four well trained operators (system operating experience: 1, 2, 2.5 and 3 years) were required to analyze the match independently (that was: two operators coded Real Madrid and the other two coded Valencia). All operators were unaware that the study was being undertaken.

Due to playing time, there were big differences between the 26 outfield players' action counts. Therefore, the agreement of their actions coded by independent operators was analyzed by dividing into three groups: (1) attacking related actions: shot, assist, key pass, through ball, cross, dribble, foul drawn, dispossessed, turnover, offside and corner won; (2) defending related actions: ball recovery, block (of shots, crosses and passes), 
challenge, tackle, clearance, interception, foul committed, corner lost, shield ball out of play, offside provoked, yellow card and red card; (3) passing and other actions: pass, throw in, aerial duel and touches. Furthermore, the inter-operator reliability of some key performance indicators (shot, dribble, pass, cross, key pass, through ball, tackle and interception, ball recovery, block (of shots, crosses and passes), clearance, card, aerial duel, corner and foul) were analyzed.

All the actions and their timing recorded by independent operators were output, transferred and analyzed in the software of Microsoft Excel 2007.

The whole data collection procedure was supported by OPTA Spain Company. Institutional ethics committee approval was obtained from the Polytechnic University of Madrid before the experimental test.

\subsection{Statistical Analysis}

Nominal variables (recorded team events and goalkeeper actions) were compared between two operators by computing the weighted kappa statistic. The method undertaken to calculate weighted kappa value was the one suggested by O'Donoghue (2010, pp. 161-164) and Robinson and O'Donoghue (2007). The agreement interpretation of kappa value was as follows: <0 less than chance agreement; 0.01-0.20 poor agreement; 0.21-0.40 fair agreement; 0.41-0.60 moderate agreement; 0.61-0.80 good agreement; 0.81-0.99 very good agreement (Altman, 1991; Viera and Garrett, 2005).

Absolute reliability values (mean, change in the mean, standardized typical error and intra-class correlation coefficient) of different types of match actions and some of the key performance indicators of outfield players were calculated using the spreadsheet developed by Hopkins (2000b). As the two teams were coded by two different operators independently, there were two groups of absolute reliability values, one for Real Madrid's players and the other for Valencia's. The results presented were the means of the two groups. The value of standardized typical error should be doubled and their levels of disagreement are as following: <0.20 trivial; 0.21-0.60 small; 0.61-1.20 moderate; 1.21-2.00 large; 2.01-4.00 very large; >4.00 extremely large (Hopkins, 2000b; Smith and Hopkins, 2011).

\section{Results}

Table 1 showed that there were 1509 events agreed by two independent operators, 855 for Real Madrid and 654 for Valencia. Details can be seen from Table 3 and Table4. The average difference of event time was $0.06 \pm 0.04$ s. The Kappa values of the two teams' events were 0.92 and 0.94 which showed a very good agreement between independent operators. 
Table 1. Agreement of Team Events Coded by Independent Operators

\begin{tabular}{ccccccc}
\hline Events & \multirow{2}{*}{$\begin{array}{c}\text { Agreed } \\
\text { Teams }\end{array}$} & \multicolumn{2}{c}{ Events Coded by Operator 1 } & \multicolumn{2}{c}{ Events Coded by Operator 2 } & Kappa \\
\cline { 3 - 6 } & Events & Total & Disagreed & Total & Disagreed & Value \\
\hline Real Madrid & 855 & 887 & 32 & 879 & 24 & 0.92 \\
Valencia & 654 & 679 & 25 & 664 & 10 & 0.94 \\
\hline
\end{tabular}

As can be seen in Table 2, there were 95 goalkeeper actions observed by both of the two operators, 42 for Iker Casillas and 53 for Diego Alves. Detailed statistics can be seen from Table 5 and Table 6. The Kappa values of goalkeeper actions were 0.92 and 0.86 which also showed very good agreement between operators.

Table 2. Agreement of Goalkeeper Actions Coded by Independent Operators

\begin{tabular}{|c|c|c|c|c|c|c|}
\hline Events & Agreed & Events $\mathrm{C}$ & y Operator 1 & Events & y Operator 2 & Карра \\
\hline Player & Events & Total & Disagreed & Total & Disagreed & Value \\
\hline Iker Casillas & 42 & 43 & 1 & 44 & 2 & 0.92 \\
\hline Diego Alves & 53 & 59 & 6 & 55 & 2 & 0.86 \\
\hline
\end{tabular}




\begin{tabular}{|c|c|c|c|c|c|c|c|c|c|c|c|c|c|c|c|c|c|c|c|c|c|c|c|c|c|c|c|c|c|c|c|c|c|c|c|c|c|c|c|c|c|c|c|c|c|c|}
\hline \multirow{2}{*}{\multicolumn{2}{|c|}{ Real Madrid }} & \multicolumn{45}{|c|}{ Operator 2} \\
\hline & & 党 & 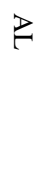 & $\mathscr{\varpi}$ & $\approx$ & $\stackrel{\square}{\sim}$ & $\begin{array}{l}\frac{\varpi}{\circ} \\
\frac{\check{2}}{\pi}\end{array}$ & $\stackrel{\square}{\Omega}$ & $\stackrel{\square}{\nabla}$ & $\widehat{\cong}$ & 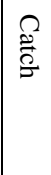 & 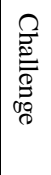 & 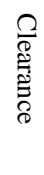 & $\approx$ & $\xi$ & $\S$ & 尺े & 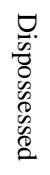 & T: & 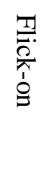 & 김 & T⿱⺌兀 & $\begin{array}{l}\stackrel{Q}{0} \\
\stackrel{\mathscr{E}}{2}\end{array}$ & 公 & 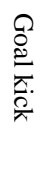 & 六 & 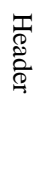 & 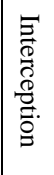 & 夌 & 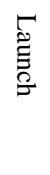 & 3 &  & 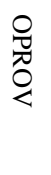 & $\stackrel{\varrho}{\Xi}$ & $\ddot{E}$ & $\begin{array}{l}\overrightarrow{0} \\
\text { T] }\end{array}$ & $\stackrel{?}{\stackrel{Z}{Z}}$ & $\begin{array}{l}\overrightarrow{0} \\
\stackrel{0}{a}\end{array}$ & 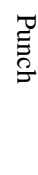 & $\begin{array}{l}\mathscr{\tilde { U }} \\
\stackrel{\tilde{C}}{2}\end{array}$ & $\begin{array}{l}\mathscr{0} \\
0 \\
0 \\
0\end{array}$ & 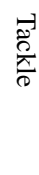 & 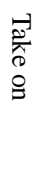 &  & 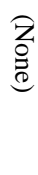 & 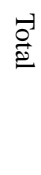 \\
\hline & AW & 15 & & & & & & & & & & & & & & & & & & & & & & & & & & & & & & & & & & & & & & & & & & & 0 & 15 \\
\hline & $\mathrm{AL}$ & & 6 & & & & & & & & & & & & & & & & & & & & & & & & & & & & & & & & & & & & & & & & & & 0 & 6 \\
\hline & SB & & & 2 & & & & & & & & & & & & & & & & & & & & & & & & & & & & & & & & & & & & & & & & & 0 & 2 \\
\hline & SS & & & & 8 & & & & & & & & & & & & & & & & & & & & & & & & & & & & & & & & & & & & & & & & 0 & 8 \\
\hline & BR & & & & & 42 & & & & & & & & & & & & & & & & & & & & & & & & & & & & & & & & & & & & & & & 5 & 47 \\
\hline & Block & & & & & & 1 & & & & & & & & & & & & & & & & & & & & & & & & & & & & & & & & & & & & & & 0 & 1 \\
\hline & $\mathrm{BC}$ & & & & & & & 0 & & & & & & & & & & & & & & & & & & & & & & & & & & & & & & & & & & & & & 0 & 0 \\
\hline 0 & BP & & & & & & & & 8 & & & & & & & & & & & & & & & & & & & & & & & & & & & & & & & & & & & & 1 & 9 \\
\hline$p$ & Card & & & & & & & & & 1 & & & & & & & & & & & & & & & & & & & & & & & & & & & & & & & & & & & 0 & 1 \\
\hline e & Catch & & & & & & & & & & 1 & & & & & & & & & & & & & & & & & & & & & & & & & & & & & & & & & & 0 & 1 \\
\hline r & Challenge & & & & & & & & & & & 1 & & & & & & & & & & & & & & & & & & & & & & & & & & & & & & & & & 0 & 1 \\
\hline $\mathrm{a}$ & Clearance & & & & & & & & & & & & 15 & & & & & & & & & & & & & & 4 & 3 & & & & & & & 2 & & & & & & & & & & 2 & 26 \\
\hline $\mathrm{t}$ & $\mathrm{CB}$ & & & & & & & & & & & & & 7 & & & & & & & & & & & & & & & & & & & & & & & & & & & & & & & 0 & 7 \\
\hline o & $\mathrm{CW}$ & & & & & & & & & & & & & & 12 & & & & & & & & & & & & & & & & & & & & & & & & & & & & & & 0 & 12 \\
\hline$r$ & $\mathrm{CL}$ & & & & & & & & & & & & & & & 5 & & & & & & & & & & & & & & & & & & & & & & & & & & & & & 0 & 5 \\
\hline & Cross & & & & & & & & & & & & & & & & 33 & & & & & & & & & & & & & & & & & & & & & & & & & & & & 4 & 37 \\
\hline 1 & Dispossessed & & & & & & & & & & & & & & & & & 21 & & & & & & & & & & & & & & & & & & & & & & & & & & & 1 & 22 \\
\hline & Error & & & & & & & & & & & & & & & & & & 1 & & & & & & & & & & & & & & & & & & & & & & & & & & 0 & 1 \\
\hline & Flick-on & & & & & & & & & & & & & & & & & & & 4 & & & & & & & 2 & & & & & & & & & & & & & & & & & & 0 & 6 \\
\hline & FD & & & & & & & & & & & & & & & & & & & & 15 & & & & & & & & & & & & & & & & & & & & & & & & 0 & 15 \\
\hline & Foul & & & & & & & & & & & & & & & & & & & & & 10 & & & & & & & & & & & & & & & & & & & & & & & 0 & 10 \\
\hline & Goal & & & & & & & & & & & & & & & & & & & & & & 1 & & & & & & & & & & & & & & & & & & & & & & 0 & 1 \\
\hline & GKKH & & & & & & & & & & & & & & & & & & & & & & & 0 & & & & & & & & & & & & & & & & & & & & & 0 & 0 \\
\hline & Goal kick & & & & & & & & & & & & & & & & & & & & & & & & 4 & & & & & & & & & & 1 & & & & & & & & & & 0 & 5 \\
\hline
\end{tabular}




\begin{tabular}{|c|c|c|c|c|c|c|c|c|c|c|c|c|c|c|c|c|c|c|c|c|c|c|c|c|c|c|c|c|c|c|c|c|c|c|c|c|c|c|c|c|c|c|c|c|c|}
\hline GKT & & & & & & & & & & & & & & & & & & & & & & & & & 8 & & & & & & & & & & & & & & & & & & & 0 & 8 \\
\hline Header & & & & & & & & & & & & & & & & & & & & & & & & & & 23 & & & & & & & & & & & & & & & & & & 0 & 23 \\
\hline Interception & & & & & & & & & & & & & & & & & & & & & & & & & & & 13 & & & & & & & & & & & & & & & & & 0 & 13 \\
\hline GKS & & & & & & & & & & & & & & & & & & & & & & & & & & & & 1 & & & & & & & & & & & & & & & & 0 & 1 \\
\hline Launch & & & & & & & & & & & & & & & & & & & & & & & & & & & & & 9 & & & & & & & & & & & & & & & 0 & 9 \\
\hline Miss & & & & & & & & & & & & & & & & & & & & & & & & & & & & & & 10 & & & & & & & & & & & & & & 0 & 10 \\
\hline OPASS & & & & & & & & & & & & & & & & & & & & & & & & & & & & & & & 3 & & & & & & & & & & & & & 0 & 3 \\
\hline OPROV & & & & & & & & & & & & & & & & & & & & & & & & & & & & & & & & 5 & & & & & & & & & & & & 0 & 5 \\
\hline Out & & & & & & & & & & & & & & & & & & & & & & & & & & & & & & & & & 64 & & & & & & & & & & & 0 & 64 \\
\hline Pass & & & & & & & & & & & & & & & & & & & & & & & & & & & & & & & & & & 443 & & & & & & & & & & 4 & 447 \\
\hline POFF & & & & & & & & & & & & & & & & & & & & & & & & & & & & & & & & & & & 3 & & & & & & & & & 0 & 3 \\
\hline PON & & & & & & & & & & & & & & & & & & & & & & & & & & & & & & & & & & & & 3 & & & & & & & & 0 & 3 \\
\hline Post & & & & & & & & & & & & & & & & & & & & & & & & & & & & & & & & & & & & & 1 & & & & & & & 0 & 1 \\
\hline Punch & & & & & & & & & & & & & & & & & & & & & & & & & & & & & & & & & & & & & & 0 & & & & & & 0 & 0 \\
\hline Save & & & & & & & & & & & & & & & & & & & & & & & & & & & & & & & & & & & & & & & 1 & & & & & 0 & 1 \\
\hline SBOP & & & & & & & & & & & & & & & & & & & & & & & & & & 2 & & & & & & & & & & & & & & 0 & & & & 0 & 2 \\
\hline Tackle & & & & & & & & & & & & & & & & & & & & & & & & & & & & & & & & & & & & & & & & & 11 & & & 0 & 11 \\
\hline Take on & & & & & & & & & & & & & & & & & & & & & & & & & & & & & & & & & & & & & & & & & & 22 & & 0 & 22 \\
\hline Throw in & & & & & & & & & & & & & & & & & & & & & & & & & & & & & & & & & & & & & & & & & & & 22 & 1 & 23 \\
\hline (None) & 0 & 1 & 0 & 0 & 0 & 0 & 0 & 0 & 0 & 0 & 0 & 0 & 0 & 0 & 0 & 0 & 0 & 0 & 0 & 0 & 0 & 0 & 0 & 0 & 0 & 0 & 3 & 0 & 0 & 0 & 0 & 0 & 0 & 4 & 0 & 0 & 0 & 0 & 0 & 0 & 0 & 2 & 0 & & 10 \\
\hline Total & 15 & 7 & 2 & 8 & 42 & 1 & 0 & 8 & 1 & 1 & 1 & 15 & 7 & 12 & 5 & 33 & 21 & 1 & 4 & 15 & 10 & 1 & 0 & 4 & 8 & 31 & 19 & 1 & 9 & 10 & 3 & 5 & 64 & 450 & 3 & 3 & 1 & 0 & 1 & 0 & 11 & 24 & 22 & 18 & 911 \\
\hline
\end{tabular}


Hands; GKT: Goalkeeper Throw; GKS: Goalkeeper Sweeper; OPASS: Offside Pass; OPROV: Offside Provoked; POFF: Player Off; PON: Player On; SBOP: Shield Ball Out of Play 


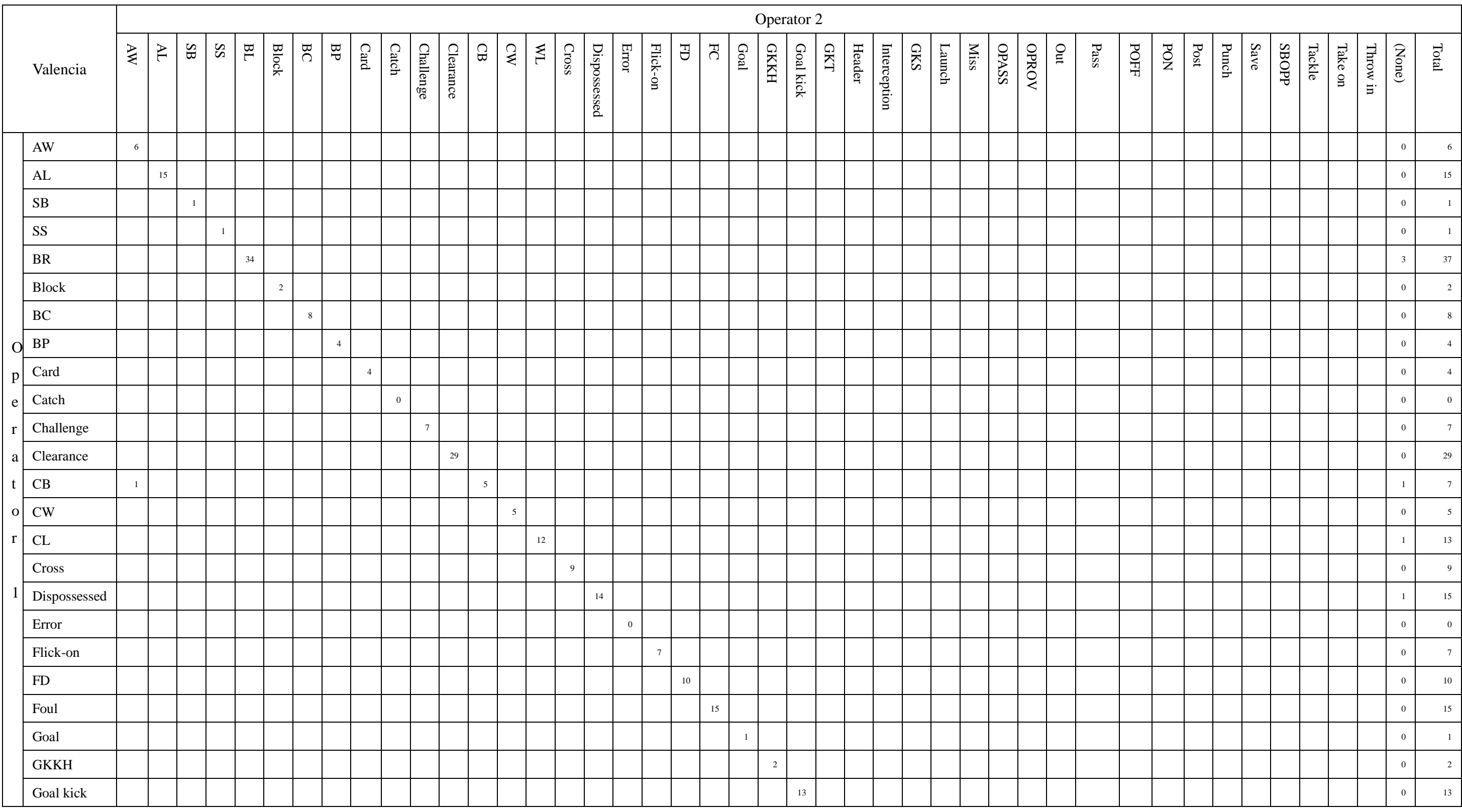




\begin{tabular}{|c|c|c|c|c|c|c|c|c|c|c|c|c|c|c|c|c|c|c|c|c|c|c|c|c|c|c|c|c|c|c|c|c|c|c|c|c|c|c|c|c|c|c|c|c|c|}
\hline GKT & & & & & & & & & & & & & & & & & & & & & & & & & 3 & & & & & & & & & & & & & & & & & & & 0 & 3 \\
\hline Header & & & & & & & & & & & & & & & & & & & & & & & & & & 6 & & & & & & & & & & & & & & & & & & 1 & 7 \\
\hline Interception & & & & & & & & & & & & & & & & & & & & & & & & & & & 10 & & & & & & & & & & & & & & & & & 0 & 10 \\
\hline GKS & & & & & & & & & & & & & & & & & & & & & & & & & & & & 2 & & & & & & & & & & & & & & & & 0 & 2 \\
\hline Launch & & & & & & & & & & & & & & & & & & & & & & & 1 & & & & & & 17 & & & & & & & & & & & & & & & 0 & 18 \\
\hline Miss & & & & & & & & & & & & & & & & & & & & & & & & & & & & & & 2 & & & & & & & & & & & & & & 0 & 2 \\
\hline OPASS & & & & & & & & & & & & & & & & & & & & & & & & & & & & & & & 5 & & & & & & & & & & & & & 0 & 5 \\
\hline OPROV & & & & & & & & & & & & & & & & & & & & & & & & & & & & & & & & 3 & & & & & & & & & & & & 0 & 3 \\
\hline Out & & & & & & & & & & & & & & & & & & & & & & & & & & & & & & & & & 64 & & & & & & & & & & & 0 & 64 \\
\hline Pass & & & & & & & & 2 & & & & 1 & & & & & & & & & & & & & & & & & & & & & & 272 & & & & & & & & & & 13 & 288 \\
\hline POFF & & & & & & & & & & & & & & & & & & & & & & & & & & & & & & & & & & & 3 & & & & & & & & & 0 & 3 \\
\hline PON & & & & & & & & & & & & & & & & & & & & & & & & & & & & & & & & & & & & 3 & & & & & & & & 0 & 3 \\
\hline Post & & & & & & & & & & & & & & & & & & & & & & & & & & & & & & & & & & & & & 0 & & & & & & & 0 & 0 \\
\hline Punch & & & & & & & & & & & & & & & & & & & & & & & & & & & & & & & & & & & & & & 2 & & & & & & 0 & 2 \\
\hline Save & & & & & & & & & & & & & & & & & & & & & & & & & & & & & & & & & & & & & & & 8 & & & & & 0 & 8 \\
\hline SBOP & & & & & & & & & & & & & & & & & & & & & & & & & & & & & & & & & & & & & & & & 0 & & & & 0 & 0 \\
\hline Tackle & & & & & & & & & & & & & & & & & & & & & & & & & & & & & & & & & & & & & & & & & 23 & & & 0 & 23 \\
\hline Take on & & & & & & & & & & & & & & & & & & & & & & & & & & & & & & & & & & & & & & & & & & 5 & & 0 & 5 \\
\hline Throw in & & & & & & & & & & & & & & & & & & & & & & & & & & & & & & & & & & & & & & & & & & & 22 & 0 & 22 \\
\hline (None) & 0 & 0 & 0 & 0 & 0 & 0 & 0 & 0 & 0 & 0 & 0 & 0 & 0 & 0 & 0 & 0 & 0 & 0 & 0 & 0 & 0 & 0 & 0 & 0 & 0 & 0 & 0 & 0 & 1 & 0 & 0 & 0 & 0 & 2 & 0 & 0 & 0 & 0 & 0 & 0 & 1 & 1 & 0 & & 5 \\
\hline Total & 7 & 15 & 1 & 1 & 34 & $2^{2}$ & 8 & 6 & 4 & 0 & 7 & 30 & 5 & 5 & 12 & 9 & 14 & 0 & 7 & 10 & 15 & 1 & 3 & 13 & 3 & 6 & 10 & 2 & 18 & 2 & 5 & 3 & 64 & 274 & 3 & 3 & 0 & 2 & 8 & 0 & 24 & 6 & 22 & 20 & 689 \\
\hline
\end{tabular}

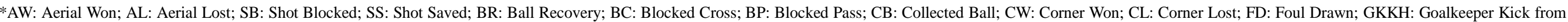
Hands; GKT: Goalkeeper Throw; GKS: Goalkeeper Sweeper; OPASS: Offside Pass; OPROV: Offside Provoked; POFF: Player Off; PON: Player On; SBOP: Shield Ball Out of Play 
Table 5. Iker Casillas's Goalkeeper Actions Coded by Independent Operators

\begin{tabular}{|c|c|c|c|c|c|c|c|c|c|c|c|c|c|c|c|c|c|c|}
\hline & & & & & & & & & & $\mathrm{rat}$ & & & & & & & & \\
\hline & ker Casillas & $\stackrel{\gtrless}{k}$ & $\underset{\nabla}{\square}$ & กี & & $\widetilde{\sigma}$ & $\beta$ & 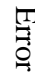 & & $\bar{\lambda}$ & 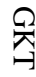 & 증 & 节 & 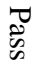 &  & 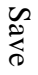 & $\begin{array}{l}\text { Zz } \\
\stackrel{8}{Z}\end{array}$ & $\stackrel{\overrightarrow{0}}{\vec{D}}$ \\
\hline & AW & 0 & & & & & & & & & & & & & & & & 0 \\
\hline & $\mathrm{BR}$ & & 3 & & & & & & & & & & & & & & & 3 \\
\hline & Catch & & & 1 & & & & & & & & & & & & & & 1 \\
\hline & Clearance & & & & & & & & & & & & & & & & & 2 \\
\hline $\mathrm{O}$ & $\mathrm{CB}$ & & & & & 7 & & & & & & & & & & & & 7 \\
\hline $\mathrm{p}$ & CL & & & & & & 1 & & & & & & & & & & & 1 \\
\hline $\mathrm{e}$ & Error & & & & & & & 1 & & & & & & & & & & 1 \\
\hline $\mathrm{r}$ & Goal Kick & & & & & & & & 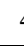 & & & & & 1 & & & & 5 \\
\hline $\mathrm{a}$ & GKTH & & & & & & & & & 0 & & & & & & & & 0 \\
\hline $\mathrm{t}$ & GKT & & & & & & & & & & 8 & & & & & & & 8 \\
\hline o & GKS & & & & & & & & & & & 1 & & & & & & 1 \\
\hline $\mathrm{r}$ & Launch & & & & & & & & & & & & 3 & & & & & 3 \\
\hline 1 & Pass & & & & & & & & & & & & & 10 & & & & 10 \\
\hline & Punch & & & & & & & & & & & & & & 0 & & & 0 \\
\hline & Save & & & & & & & & & & & & & & & 1 & & 1 \\
\hline & (None) & & & & & & & & & & & & & 1 & & & & 1 \\
\hline & Total & 0 & 3 & 1 & & 7 & 1 & 1 & 2 & c & 8 & 1 & 3 & 12 & 0 & 1 & 0 & 45 \\
\hline
\end{tabular}

*AW: Aerial Won; BR: Ball Recovery; CB: Collected Ball; CL: Corner Lost; GKKH: Goalkeeper Kick from

Hands; GKT: Goalkeeper Throw; GKS: Goalkeeper Sweeper 
Table 6. Diego Alves's Goalkeeper Actions Coded by Independent Operators

\begin{tabular}{|c|c|c|c|c|c|c|c|c|c|c|c|c|c|c|c|c|c|c|}
\hline & & & & & & & & & & rat & & & & & & & & \\
\hline & iego Alves & $\stackrel{8}{\&}$ & $\underset{\nabla}{\varpi}$ & $\begin{array}{l}\widetilde{\tilde{*}} \\
\stackrel{\tilde{\sigma}}{\sigma}\end{array}$ & $\stackrel{\Omega}{\nexists}$ & 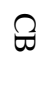 & $\Omega$ & ب) & $\begin{array}{l}\Omega \\
0 \\
0\end{array}$ & 元 & $\Omega$ & 忩 & 芯 & Dे & $\begin{array}{l}\vec{Z} \\
\underline{\Xi} \\
\end{array}$ & 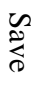 & Ż & $\overrightarrow{\stackrel{0}{0}}$ \\
\hline & AW & 0 & & & & & & & & & & & & & & & & 0 \\
\hline & BR & & 0 & & & & & & & & & & & & & & & 0 \\
\hline & Catch & & & 0 & & & & & & & & & & & & & & 0 \\
\hline & Clearance & & & & 2 & & & & & & & & & & & & & 2 \\
\hline $\mathrm{O}$ & $\mathrm{CB}$ & 1 & & & & 5 & & & & & & & & & & & 1 & 7 \\
\hline o & CL & & & & & & 2 & & & & & & & & & & 1 & 3 \\
\hline $\mathrm{e}$ & Error & & & & & & & 0 & & & & & & & & & & 0 \\
\hline $\mathrm{r}$ & Goal Kick & & & & & & & & 13 & & & & & & & & & 13 \\
\hline $\mathrm{a}$ & GKKH & & & & & & & & & 2 & & & & & & & & 2 \\
\hline $\mathrm{t}$ & GKT & & & & & & & & & & 3 & & & & & & & 3 \\
\hline o & GKS & & & & & & & & & & & 2 & & & & & & 2 \\
\hline$r$ & Launch & & & & & & & & & 1 & & & 12 & & & & & 13 \\
\hline 1 & Pass & & & & & & & & & & & & & 2 & & & 2 & 4 \\
\hline & Punch & & & & & & & & & & & & & & 2 & & & 2 \\
\hline & Save & & & & & & & & & & & & & & & 8 & & 8 \\
\hline & (None) & & & & & & & & & & & & & & & & & 0 \\
\hline & Total & 1 & 0 & 0 & 2 & 5 & 2 & 0 & 13 & 3 & 3 & 2 & 12 & 2 & 2 & 8 & 4 & 61 \\
\hline
\end{tabular}

*AW: Aerial Won; BR: Ball Recovery; CB: Collected Ball; CL: Corner Lost; GKKH: Goalkeeper Kick from Hands; GKT: Goalkeeper Throw; GKS: Goalkeeper Sweeper

As can be seen from Table 7, standardized typical errors of different types of individual outfield players' match actions coded by independent operators varied from 0.08 to 0.21 , while intra-class correlation coefficients ranged from 0.96 to 0.99 which all showed high levels of reliability.

Table 7. Reliability of Individual Outfield Players' Match Actions Coded by Independent Operators

\begin{tabular}{lcccc}
\hline Indicators & Mean $\pm \mathrm{SD}$ & $\begin{array}{l}\text { Change in the mean } \\
\pm \text { confidence limits }\end{array}$ & $\begin{array}{l}\text { Standardized } \\
\text { typical error }\end{array}$ & $\begin{array}{l}\text { Intra-class } \\
\text { correlation } \\
\text { (ICC) }\end{array}$ \\
\hline Attacking Related Actions & $7.2 \pm 4.8$ & $-0.23 \pm 0.36$ & 0.16 & 0.98 \\
Defending Related Actions & $9.5 \pm 6.0$ & $-0.46 \pm 0.61$ & 0.21 & 0.96 \\
Passing and Other Actions & $33.3 \pm 18.2$ & $-0.19 \pm 0.80$ & 0.09 & 0.99 \\
Total Actions & $49.9 \pm 24.4$ & $-0.88 \pm 1.97$ & 0.08 & 0.99 \\
\hline
\end{tabular}

*Confidence limits of standardized typical error are the factors $\times / \div 1.30$ 
Table 8 showed the absolute reliability test results of some key performance indicators of individual outfield players. Standardized typical errors ranged from 0 to 0.37 , intra-class correlation coefficients varied from 0.88 to 1.00 which also showed high levels of reliability.

Table 8. Reliability of Individual Outfield Players' Key Performance Indicators Coded by Independent Operators

\begin{tabular}{lcccc}
\hline Indicators & Mean $\pm \mathrm{SD}$ & $\begin{array}{c}\text { Change in the mean } \\
\pm \text { confidence limits }\end{array}$ & $\begin{array}{c}\text { Standardized } \\
\text { typical error }\end{array}$ & $\begin{array}{c}\text { Intra-class } \\
\text { correlation } \\
\text { (ICC) }\end{array}$ \\
\hline Shots & $1.0 \pm 1.3$ & $0.00 \pm 0.00$ & 0.00 & 1.00 \\
Dribbles & $1.1 \pm 1.5$ & $0.08 \pm 0.22$ & 0.23 & 0.96 \\
Crosses & $1.7 \pm 2.2$ & $-0.15 \pm 0.26$ & 0.17 & 0.98 \\
Key Passes & $1.0 \pm 1.1$ & $0.00 \pm 0.00$ & 0.00 & 1.00 \\
Through Balls & $0.4 \pm 0.7$ & $-0.04 \pm 0.12$ & 0.19 & 0.98 \\
Corners Won & $0.7 \pm 1.0$ & $0.00 \pm 0.00$ & 0.00 & 1.00 \\
Fouls Drawn & $0.9 \pm 0.7$ & $0.00 \pm 0.14$ & 0.22 & 0.92 \\
Lost Balls & $1.3 \pm 1.4$ & $0.04 \pm 0.26$ & 0.24 & 0.90 \\
\hline Tackles & $1.4 \pm 1.2$ & $-0.04 \pm 0.20$ & 0.31 & 0.91 \\
Interceptions & $1.1 \pm 1.2$ & $0.12 \pm 0.28$ & 0.37 & 0.88 \\
Ball recoveries & $3.0 \pm 2.4$ & $-0.31 \pm 0.42$ & 0.36 & 0.88 \\
Blocks & $1.0 \pm 1.1$ & $0.00 \pm 0.20$ & 0.33 & 0.90 \\
Clearances & $1.8 \pm 2.1$ & $-0.35 \pm 0.42$ & 0.33 & 0.90 \\
Corners Lost & $0.5 \pm 0.9$ & $0.04 \pm 0.25$ & 0.37 & 0.90 \\
Fouls & $1.0 \pm 0.8$ & $0.00 \pm 0.16$ & 0.37 & 0.88 \\
Cards & $0.2 \pm 0.4$ & $0.00 \pm 0.00$ & 0.00 & 1.00 \\
\hline Passes & $25.1 \pm 13.2$ & $-0.54 \pm 0.57$ & 0.09 & 0.99 \\
Aerial Duels & $1.6 \pm 2.4$ & $0.04 \pm 0.17$ & 0.10 & 0.99 \\
\hline Confidence & $1 \mathrm{c}$ & & & \\
\hline
\end{tabular}

*Confidence limits of standardized typical error are the factors $\times / \div 1.30$

\section{Discussion}

The high Kappa values, high intra-class correlation coefficients and low standardized typical errors show a high level inter-operator reliability using the OPTA Client System to collect players' and teams' match performance statistics. The strong agreement of observed actions by independent operators suggests that the human error in tracking live match actions with the system is extremely limited.

Similar to the ProZone MatchViewer system, the high inter-operator reliability of the OPTA Client System can also be attributed to the rigid operational event definitions and the high quantity of strict user training (Bradley et al., 2007). Although it is believed that precise operational definition wordings may not guarantee good reliability, but it is essential for the system operators to fully understand the variables 
and/or indicators (O'Donoghue, 2007). Obviously, OPTA do really well in these aspects during the months' time of training of operators. New users of the system are required to learn thoroughly definitions of all possible happening pitch actions and to get full awareness of coding these actions. They are also offered sufficient practice imitating live match situations using the "test servers" (OPTA, 2012).

As stated by Bradley et al. (2007), it is necessary to indentify the occasions where generate the disagreements of independent operators to produce the reliability statistics. Just like what has been found by Bradley et al. (2007), the disagreed events mainly came from the misrecognizing of individual players. For example, when most of the players stand in the penalty area challenging for a corner, it is not so easy to differentiate those who touched the ball in seconds of time. The second mostly arisen disagreements are from operators' different understandings of some definitions of actions, especially for defending actions. As can be seen from Table 7 and Table 8, the intra-class correlation coefficients of defending related actions and indicators are the lowest, while their standardized typical errors are the highest. Statistically, this could be because of their relatively smaller counts compared to other actions and indicators. While technically, there could be more possibilities for the operator to understand differently their definitions. For instance, when a defender headed a ball out of the area under the challenge of an attacking player, and the ball is received by one of his teammates. The header could be defined as "aerial won", "clearance", and/or "pass", while different operators would have different choice.

\section{Conclusion}

The current study demonstrated that the data gathering method used by OPTA Sportsdata (OPTA Client System) has a high level inter-operator reliability. The statistics generated from the system could be used validly for academic research, public publications and coaching purposes. Meanwhile, the reliability could be further strengthened by potential advanced techniques of tracking players and more specific definitions of some defending match actions.

\section{Acknowledgements}

The first author is funded by the China Scholarship Council (CSC) from the Chinese Ministry of Education. Additionally, the authors would like to express sincere gratitude to the Company of OPTA Spain. The data supports from their staves Jordi Alvaro, David Perales, Torres Marcial, Alberto Salvador, Alvaro Jimenez, Hector Alonso and Mauricio Jimenez are highly appreciated. The suggestions and comments given by Doctor Peter O’Donoghue to improve the article were also appreciated. 


\section{Reference}

Altman, D.G. (1991). Practical Statistics for Medical Research. London: Chapman \& Hall.

Bateman, R. (12 May 2010). BLOG Opta's Event Definitions. In OPTASPORTS. Retrieved October 2, 2012, from http://optasports.com/news-area/blog-optas-event-definitions.aspx.

Berg, K.E., and Richard, W. (2008). Essentials of research methods in health, physical education, exercise science, and recreation. Wolters Kluwer Health.

Bradley, P., O'Donoghue, P., Wooster, B., and Tordoff, P. (2007). The reliability of ProZone MatchViewer: a video-based technical performance analysis system. International Journal of Performance Analysis in Sport. 7, 117-129.

Bradley, P. S., Carling, C., Archer, D., Roberts, J., Dodds, A., Di Mascio, M., ... and Krustrup, P. (2011). The effect of playing formation on high-intensity running and technical profiles in English FA Premier League soccer matches. Journal of Sports Sciences. 29, 821-830.

Carling, C., Bloomfield, J., Nelsen, L., and Reilly, T. (2008). The role of motion analysis in elite soccer contemporary performance measurement techniques and work rate data. Sports Medicine. 38, 839-862.

Dellal, A., Chamari, K., Wong, D. P., Ahmaidi, S., Keller, D., Barros, R., ... and Carling, C. (2011). Comparison of physical and technical performance in European soccer match-play: FA Premier League and La Liga. European Journal of Sport Science. 11, 51-59.

Di Salvo, V., Baron, R., Tschan, H., Calderon Montero, F. J., Bachl, N., and Pigozzi, F. (2007). Performance characteristics according to playing position in elite soccer. International journal of sports medicine. 28, 222.

Downing, S. M. (2004). Reliability: on the reproducibility of assessment data. Medical Education. 38, 1006-1012.

Gómez M, Álvaro J and Barriopedro MI (2009a). Behaviour patterns of finishing plays in female and male soccer. Kronos: la revista científica de actividad física y deporte. 8: 15-24. (In Spanish: English abstract).

Gómez M, Álvaro J and Barriopedro MI (2009b). Differences in playing actions between men and women in elite soccer teams. In A Hökelmann, K White, $\mathrm{P}$ O’Donoghue (eds): Current trends in performance analysis. World Congress of Performance Analysis of Sports VIII. Magdebourg: Shaker Verlag, pp 50-51.

Gómez, M. A., Gómez-Lopez, M., Lago, C., and Sampaio, J. (2012). Effects of game location and final outcome on game-related statistics in each zone of the pitch in professional football. European Journal of Sport Science. 12, 393-398.

Hayen, A., Dennis, R. J., and Finch, C. F. (2007). Determining the intra-and inter-observer reliability of screening tools used in sports injury research.

Journal of Science and Medicine in Sport. 10, 201-210.

Hopkins, WG. (2000a). Measures of Reliability in Sports Medicine and Science. Sports Medicine. 30, 1-15. 
Hopkins, WG. (2000b). Reliability for consecutive pair of trials (Excel Spreadsheet). In: A new view of Statistics. Sportsci.org: Internet Society for Sport Science. sportsci.org/resource/stats/xrely.xls

Hughes, M., Evans, S. and Wells, J. (2001). Establishing normative profiles in performance analysis. International Journal of Performance Analysis of Sport.1, 4-27.

Hughes, M. D., and Bartlett, R. M. (2002). The use of performance indicators in performance analysis. Journal of sports sciences. 20, 739-754.

Hughes, M., Cooper, S.M. and Nevill, A. (2004). Analysis of notation data: reliability. In Notational analysis of sport, 2nd Edition (Edited by M. Hughes and I.M. Franks). London: Routledge, 189-204.

Lago, C., Casais, L., Dominguez, E., and Sampaio, J. (2010). The effects of situational variables on distance covered at various speeds in elite soccer. European Journal of Sport Science. 10, 103-109.

Lago-Peñas, C., Lago-Ballesteros, J., Dellal, A., and Gómez, M. (2010). Game-related statistics that discriminated winning, drawing and losing teams from the Spanish soccer league. Journal of Sports Science and Medicine. 9, 288-293.

Lames, M., and McGarry, T. (2007). On the search for reliable performance indicators in game sports. International Journal of Performance Analysis in Sport. 7, 62-79.

Robinson, G., and O'Donoghue, P. (2007). A weighted kappa statistic for reliability testing in performance analysis of sport. International Journal of Performance Analysis in Sport. 7, 12-19.

Smith, T. B., and Hopkins, W. G. (2011). Variability and predictability of finals times of elite rowers. Medicine Science Sports Exercise. 43, 2155-60.

Oberstone, J. (2009). Differentiating the top English premier league football clubs from the rest of the pack: Identifying the keys to success. Journal of Quantitative Analysis in Sports. 5.

Oberstone, J. (2010). Comparing English Premier League Goalkeepers: Identifying the Pitch Actions that Differentiate the Best from the Rest. Journal of Quantitative Analysis in Sports. 6.

Oberstone, J. (2011). Comparing Team Performance of the English Premier League, Siere A and La Liga. Journal of Quantitative Analysis in Sports. 7.

O’Donoghue, P.G. (2006). Performance indicators for possession and shooting in international netball. In Performance Analysis of Sport 7 (Edited by H. Dancs, M. Hughes and P.G. O’Donoghue). Cardiff: CPA UWIC Press, 459-467.

O’Donoghue, P. (2007). Reliability Issues in Performance Analysis. International Journal of Performance Analysis in Sport.7, 35-48.

O’Donoghue, P. (2010). Research Methods for Sports Performance Analysis. Routledge.

OPTA. (2012). Definiciones OPTA and Pack Trainning 2012/13. OPTA Sportsdata.

Valter, D. S., Collins, A., McNeill, B. and Cardinalle, M. (2006). Validation of Prozone ${ }^{\circledR}$ : a new video based performance analysis system. International 
Journal of Performance Analysis of Sport. 6, 108-119.

Viera, A. J., and Garrett, J. M. (2005). Understanding inter-observer agreement: the kappa statistic. Family Medicine. 37, 360-363. 